\title{
Automobile Collision Warning and Identification System using Visible Light and Wi-Fi Communication
}

\author{
Rehan Ahmad Khan, Agastya Gogoi, Rahul Srivastava, Shubham Kumar Tripathy, \\ S.Manikandaswamy
}

\begin{abstract}
This paper introduces a vehicle-to-vehicle (V2V) communication system based on visible light communication technology. A vehicle will transmit the data continuously to another vehicle in front of it using head light and the data is stored in the Secure Digital (SD) Card in comma separated value for future reference in case of emergency at the same time the data is stored in the cloud server for government reference for locate the most accident areas. Nowadays, people readily use internet in their day-to-day activities to accomplish their task by means of wireless or wired network. As users are increasing manifold, data transmission rate consequently decreasing. However, Wi-Fi imparts data rate of 150Mbps as per IEEE 802.11n, this speed is still not enough to suffice the needs of a user. Considering this, Visible light communication concept has been introduced. In this project, a comparative and analytic study about the speed of visible light and Wi-Fi communication is being done and also reduction of network jamming problem due to increasing users demand is also being done.
\end{abstract}

Index Terms: vehicle-to-vehicle (V2V), LED, Wi-Fi, SD card, Visible light communication

\section{INTRODUCTION}

The future of road travel is Vehicle to Vehicle (V2V) communication, which can prevent accidents and increase accountability of misdemeanour on road. Vehicle to vehicle communication is the wireless transmission of data between vehicles in real time and at very high speeds, as in [1].

The aim is to assist vehicles interact using Visible Light technology. As in [2],[3], it is a wireless optical networking technology that uses IEEE standards for data transmission and uses photo detectors as receiver. A system with vehicle to vehicle communication using visible light is proposed in the model because visible light communication is the fastest means of communication and at the same time a Black-Box system is devised to identify when,

Revised Version Manuscript Received on Jun 20, 2019.

Rehan Ahmad Khan, Electronics and Communication, SRM Institute of Science and Technology, Kanchipuram, India

Agastya Gogoi, Electronics and Communication, SRM Institute of Science and Technology, Kanchipuram, India.

Rahul Srivastava, Electronics and Communication, SRM Institute of Science and Technology, Kanchipuram, India.

Shubham Kumar Tripathy, Electronics and Communication, SRM Institute of Science and Technology, Kanchipuram, India

S. Manikandaswamy, Electronics and Communication, SRM Institute of Science and Technology, Kanchipuram, India where and how the fault is occurred during accidents. By means of such authenticated wireless communication system, security encryption becomes more trustworthy.

In current generation systems there have been systems proposed for vehicle to vehicle communication which is mainly to stop the incoming vehicle from colliding with the other vehicle. This system has many flaws since the incoming vehicle's speed is not being considered as for a small distance the vehicle can't be abruptly stopped, as in [4].

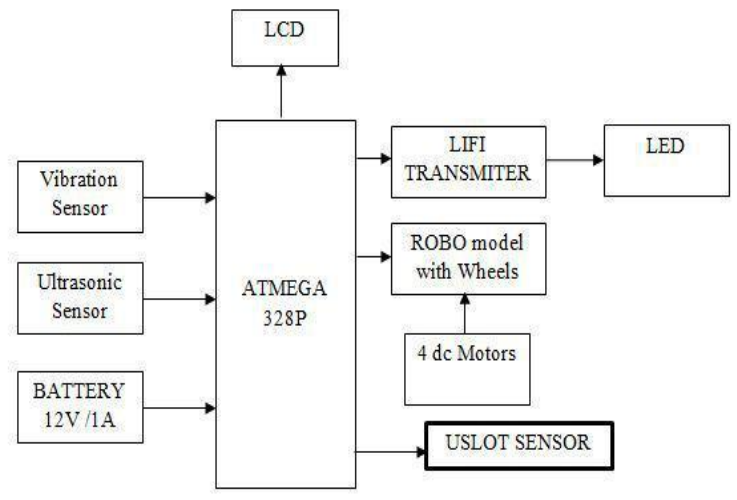

Fig.1. Transmitter

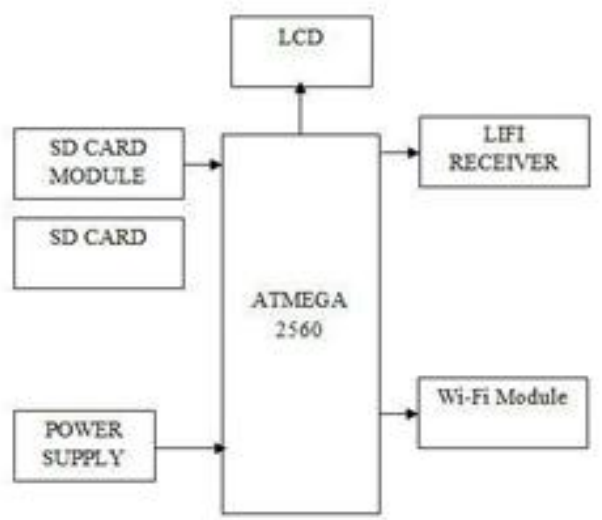

Fig.2. Receiver

The vehicle's headlights can act as transmitter and receiver can be fitted on backside of the vehicle. In the event of collision, data can be transmitted from headlight of the culprit vehicle to the victim vehicle. Vehicle registration number will also be transmitted. This information is kept securely inside the Black Box of the car.

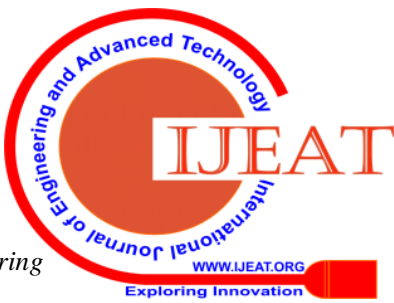


This data can be retrieved by police using Wi-Fi to catch the culprit of the collision. This system also incorporates Bluetooth services which is connected with the driver's phone. This in turn uses phone's GPS and GSM services to locate the victim which helps emergency services to be contacted.

\section{HARDWARE SETUP}

The proposed system is made using various hardware such as Arduino uno, Arduino mega, Bluetooth module, motor driver, Liquid Crystal Display (LCD), Wi-Fi module, GSM module and various sensors for performing various checks on automobile collision and data transfer. Salient features of proposed system:-

- Sensors are connected to analog and digital pins of Arduino.

- LCD panel connected to the Pulse Width Modulation (PWM) port.

- Wi-Fi module connected to $3 \mathrm{v} 3$ port of the Arduino board for $3 \mathrm{v}$ supply to the module and to the communication ports (TX,RX).

- Motor driver is connected to PWM port of the Arduino board.

\section{A. Motor Driver (L293D)}

Motor driver is used for locomotion of vehicles on road. L293D is an integrated circuit (IC) with dual H-bridge that acts as a motor driver. These integrated chips behave as current amplifiers and they amplify the incoming signal to a high current signal. This signal is used to drive the motors.

The circuit is usually operated in common mode of operation in which both motors can be driven simultaneously. One motor is controlled by input logic at pins $2 \& 7$ and enable pin 1 as high. The other one is

controlled using logic pins $10 \& 15$ and enable pin 9 as high. The following input logics are used to operate it:

- $\quad$ 01-Clockwise direction

- 10- Anticlockwise direction

- 00 or $11-$ Stop

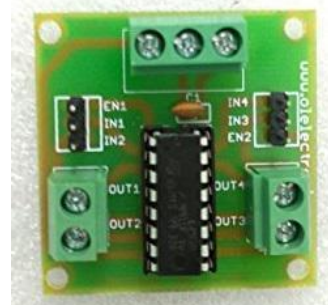

Fig.3. Motor Driver

Thus, enable pin is needed to make the drivers work. If enable input is low, the output take high impedance state and motors receive no signal.

\section{B. Ultrasonic Sensor (HC-SR04)}

Ultrasonic sensor is used to measure distance. It has two pins namely echo and trigger. It can detect any object in the range of $2 \mathrm{~cm}$ to $4 \mathrm{~m}$. It emits an ultrasound at 40,000 Hertz from the trigger pin. If there is any obstacle in its path, the ultrasound reverts back and is detected by the echo pin. The distance is then calculated by the module using the speed of sound and time required to travel the distance.

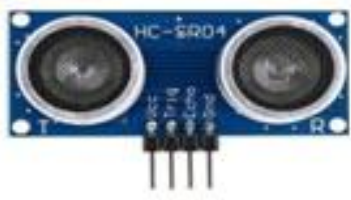

Fig.4.Ultrasonic sensor

\section{U slot sensor $($ H2010)}

$\mathrm{U}$ slot sensor module is a high-quality infrared Counter module and is integrated with LM393 chip. It contains both IR LED and IR photodetector in single package. It is used for industry counting and motor speed testing. LED light is used to show output, when object is detected, LED switches off. LED indication output is directly interfaced to a microcontroller. The output is given in digital format of 0 or 1 . Output update is very fast since it is optical. Thus it is used to calculate the rpm of the vehicle.

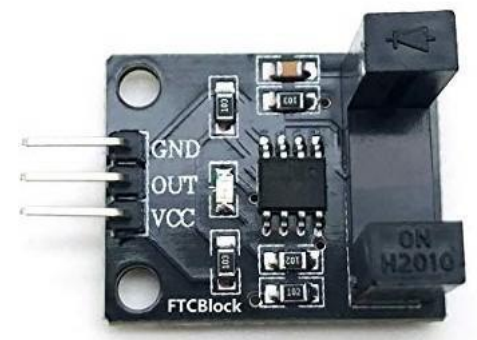

Fig.5. U slot sensor

\section{Collision sensor ( $S W 420)$}

The other name for collision sensor is piezoelectric sensor. Piezoelectric effect is the phenomena which measures changes in various parameters such as pressure, strain, temperature, acceleration or force by transforming them into electric charge. The sensors are very reliable and have a maximum vibration reading of $294000 \mathrm{~m} / \mathrm{s}^{2}$.

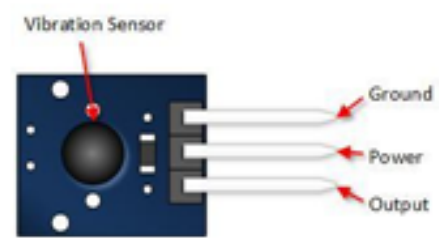

Fig.6. Collision sensor

At times, sensors can be sensitive for two or more physical quantity. For example, when exposed to vibrations, pressure sensor can depict false information. That is why, acceleration compensated elements is to be used in summation with pressure sensing components. These sensors are of importance for the integrity of the system, as this is the sensor which detects the occurrence of accident.

\section{E. Li--Fi Transmitter}

Li-Fi Transmitter is the module consisting of mainly the LED and its driving circuitry. This module will help in the transmission of data from one vehicle to the other. The LED in the module acts as the headlight of a vehicle, which then transmits light, along with the data of the user to the other vehicle which in turn has a Li-Fi Receiver, namely a phototransistor which converts the data into photocurrent. The light transmitted from the Li-Fi Transmitter circuitry acts as a medium for the data to be sent from one vehicle to the other.

Published By: 
The LED used in the system has a $12 \mathrm{~V}$ input voltage and have its luminous flux to be 600 lumen, which is standard for a headlight in a vehicle.

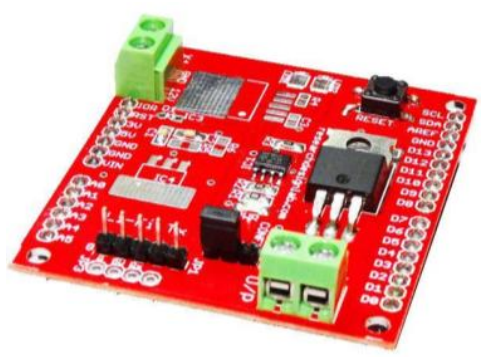

Fig.7. Li-Fi transmitter

\section{F. Li-Fi Receiver}

Li-Fi Receiver chiefly contains a phototransistor and a transimpedance amplifier circuit. The light from the LED transmitted from the transmitter is received by the phototransistor, which then converts into photocurrent. The photocurrent is then converted into photo-voltage by the trans-impedance circuit, which can be used for the receiver circuitry. The light from the transmitter is actually embedded with data from the system of the transmitter vehicle. The data is then retrieved by the receiving vehicle which is then used for different purposes.

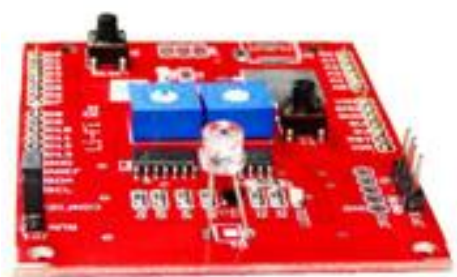

Fig.8 Li-Fi Receiver

\section{G. LCD panel}

The LCD1602 Parallel LCD is a display used easily with Arduino due to ease of set up and programming. It has a 16 by 2 character line display and sports a high contrast feature. It uses green light as backlight. The display uses IIC/I2C interface to reduce the number of pins used on the Arduino board. The following pins are included:

- Data Pins (D0-D7)

- Display contrast pin (Vo)

- Power supply pins (+5V and Gnd)

- LED Backlight (Bklt+ and BKlt-)

- $\operatorname{Read} /$ Write $(\mathrm{R} / \mathrm{W})$ pin

- Enable pin

- Register select (RS) pin

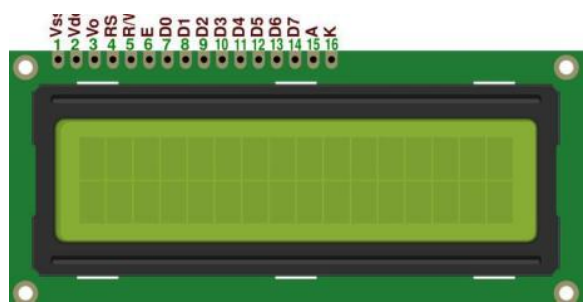

Fig.9. LCD panel

The data pins are high when data is being read or written. LCD panel here displays distance, rpm of the vehicle. As the collision occurs, the panel shows vibration sensor value as 1 and thereby displays "Accident Detect", consequently "Data send" displays on LCD at receiver side.

\section{Wi-Fi module (ESP8266)}

The ZG2100M module is a less power consuming $802.11 \mathrm{~b}$ implementation device. It provides simple and cheaper way to provide Wi-Fi connectivity to embedded devices. It contains radio frequency components, baseband and MAC address. It can easily be interfaced with 8 or 16 bit microcontrollers. It also supports latest security standards such as WPA and WPA2.

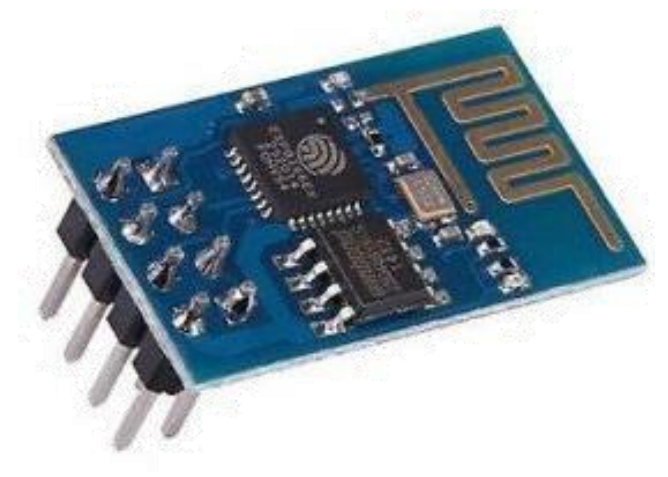

Fig.10. Wi-Fi module

Wi-Fi module is being used to enable data transfer to cloud server as and when the network available. The data being speed, date, time, rpm and registration number of vehicle. In this module, pin 1 and pin 5 are connected with transmitter and receiver of Arduino port. Pin 2 and Pin 4 are interconnected. $3.3 \mathrm{~V}$ supply is provided from Arduino at pin 4 of module, while pin 8 is grounded.

\section{A. GPS module}

GPS is a system which is used to identify location.

GPS is a satellite based radio navigation system. It sends time $\&$ geo location to any GPS receiver on earth. The condition being that, there should be an unobstructed line of sight path to four or more GPS satellites in real time. This system comprises satellites, receivers and ground stations. Like, stars in constellations, satellites exist and it is knows at any particular time, where satellites are going to be present. The location of a user can be calculated using distance from each satellite in real time.

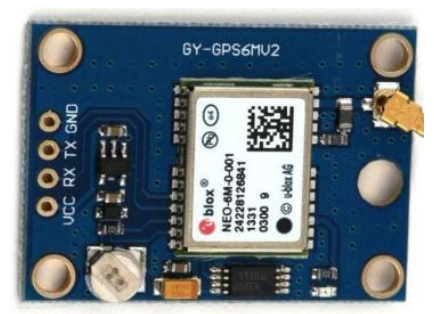

Fig.11. GPS module

This module here is used to locate driver's phone address. It senses longitude, latitude and location of driver at the time of collision.

\section{B. Bluetooth module (HC-05)}

$\mathrm{HC}-05$ module follows Bluetooth serial port protocol.. It is designed for transparent wireless serial connection setup. Range of Bluetooth module is from $3.3 \mathrm{~V}$ to $5 \mathrm{~V}$. Driver's

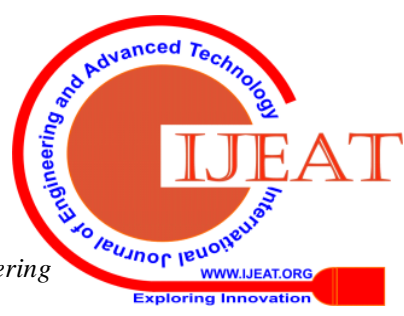


phone is connected to the system through Bluetooth. Bluetooth module is use to enable Bluetooth services. As the collision occur, a message is send to the emergency contacts notifying the message "Need Help, come fast" as well as the location of the driver.

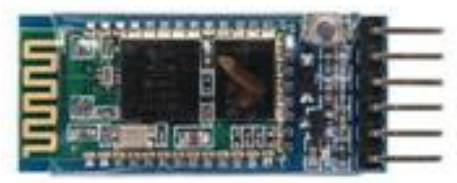

Fig.12. Bluetooth module

\section{SD card module}

The system incorporates 'Black box' based mechanism similar to airplanes. The data transfer will be in two scenarios. In first scenario, if the vehicle comes closer to it, but not collide with the other vehicle.In such case data transfer to SD card will take place, but as the collision didn't happen, data will be erased automatically. In second scenario, if the vehicle comes nearer to the vehicle, and collide with the other vehicle, the data transfer to SD card will take place and will be stored permanently in it.

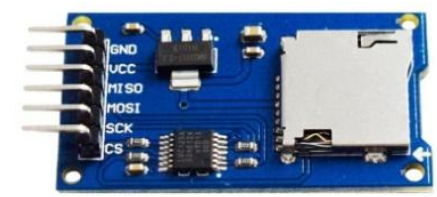

Fig.12. SD card module

\section{WORKING MODEL}

This working model comprises of two cars, former is to demonstrate the front side of the car, while the later one is to demonstrate the rear side of the car.

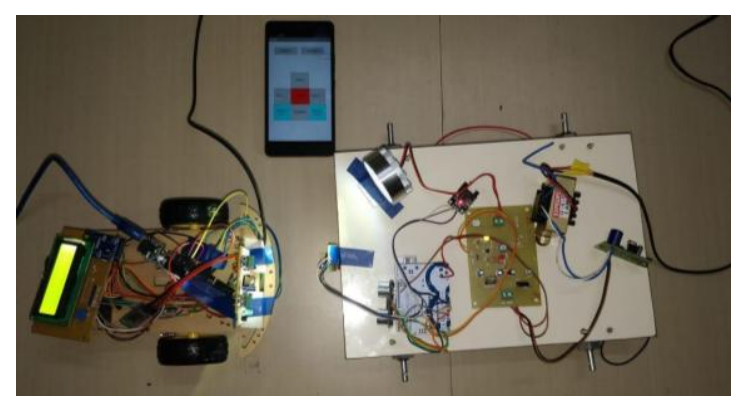

Fig.13. Automobile

\section{A. LCD initialization}

As and when power supply to the system starts, the LCD attached to the model is initialized.

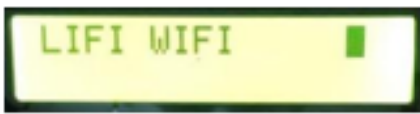

Fig.14. LCD start

B. Before collision
When the rear car comes close to the front car to the extent as set by the user, then LCD starts showing the distance and speed of the rear car on the front car panel in order to alert the navigator.

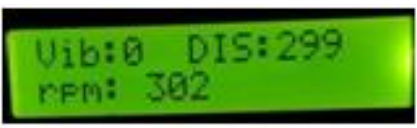

Fig.15. Prior to collision

\section{Collision}

When the rear car comes close to the front car to the extent as set by the user, then LCD starts showing the distance and speed of the rear car on the front car panel in order to alert the navigator.

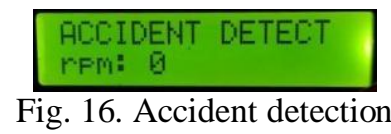

\section{After Collision}

Aftermath of the collision, for immediate medical and administration help, drivers phone is connected to system Bluetooth, and will send data to emergency contacts indicating location of the driver asking for help.

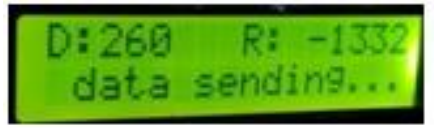

Fig. 17. Data transfer

\section{E. Mobile Application}

Mobile application is developed to enable automatic data transfer indicating location, latitude and longitude to emergency contacts.
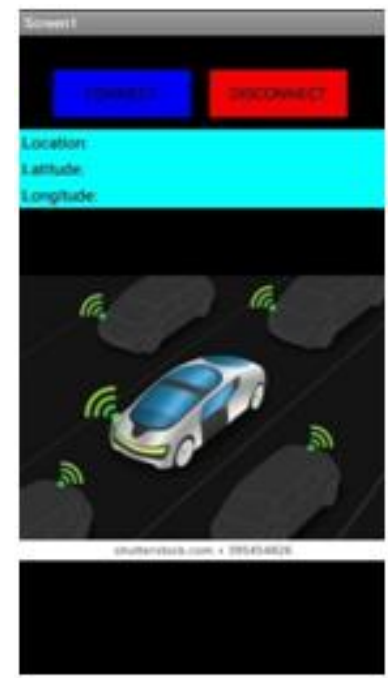

Fig. 18. Mobile App

\section{F. Website}

As vehicles data is being sent on cloud platform, to prevent data theft, a password protected web platform is developed which can only be accessed by concerned authorities in the event of need.

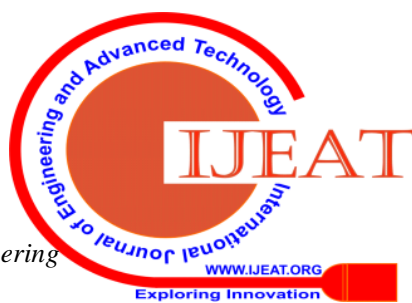




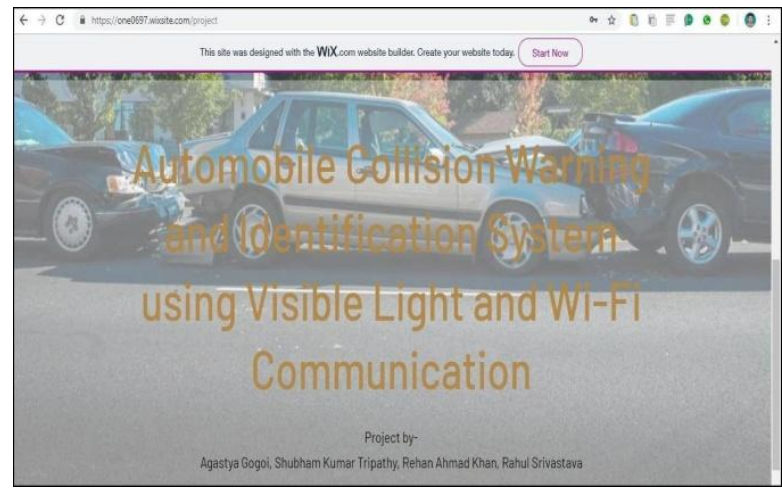

Fig.19. Website

\section{G. Cloud data representation}

Website will show graphically the track record of vehicle since its inception on road.

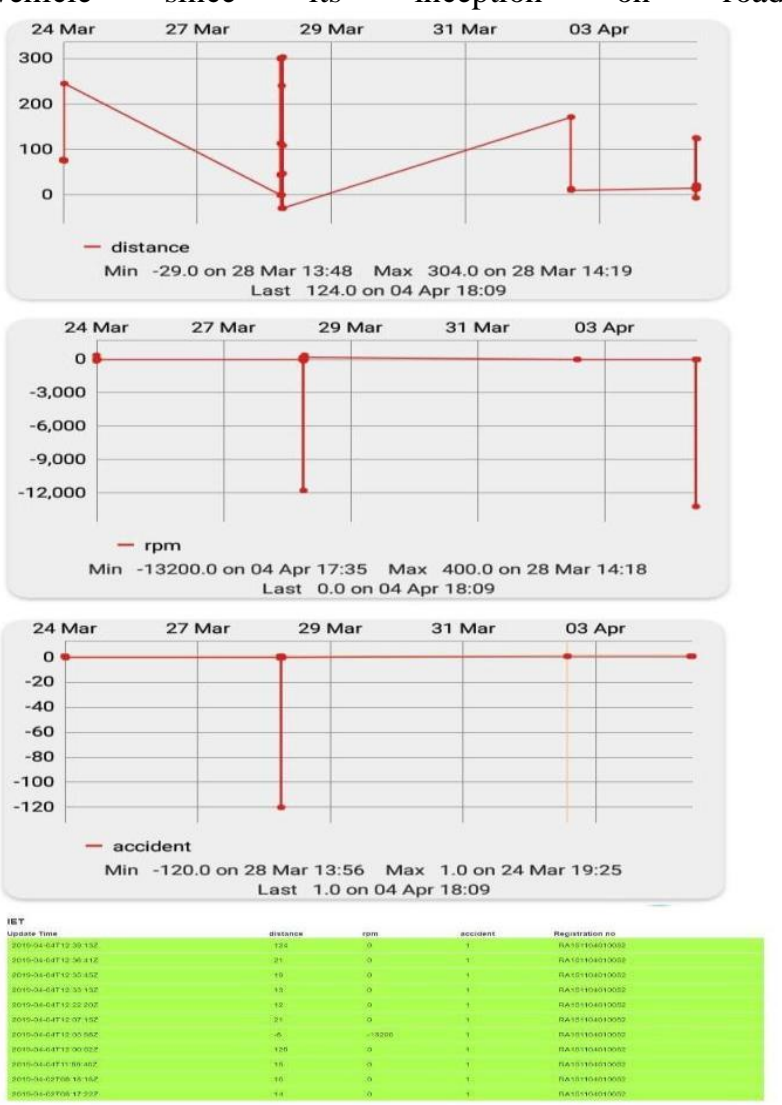

Fig.20. Thinkview data

\section{CONCLUSION}

The paper is introduced in a way to increase accountability to reduce road accidents. It also introduces a vehicle-to-vehicle (V2V) communication system based on visible light communication. The prototype successfully uses visible light to transmit data from one vehicle to other. The data is stored in the Black Box securely as comma separated value for future reference and at the same time is uploaded to the cloud. The data uploaded to the cloud is easily accessible by the police and is password protected to prevent unauthorized access. The system also helps to determine the location and time of the accident.This is done by means of GPS and GSM services present in mobile phones.These services are accessed by a mobile application, created specifically for this system. By the use of GSM service, the system can automatically messages to the emergency contacts as and when accident occurs. The proposed prototype shows fine potential for future vehicle communications scheme using visible light as the fastest medium, as in [5]. Thereby the system increases accountability to road accidents. Hence, gradually lower the rate of these accidents in the near future. In future, this work can be more effective with the use of camera sensor. Camera sensor, as in [6] can be used to determine the accused vehicle number or even the accuser himself. In the proposed existing system data transfers between the vehicles. For future use, images or videos can also be transferred between the cars. For this purpose a higher level of complexity is needed which can be done by using a complex microprocessor as it requires digital signal processing.

Another significant enhancement would be the use of onboard sensors, already present in the car for the purpose of inter-vehicular communication rather than using a new set of sensors, as in the proposed system.

In future this prototype can be made fully using photonic IC, which will incur following benefits to the system -

1. It makes the whole system more discrete, compact, and also helps in providing a high performance.

2. The chip can also be integrated with basic electronic circuits which makes it capable for more functions.

\section{REFERENCES}

1. Noof Al Abdulsalam, Raya Al Hajri, Zahra Al Abri, Zainab Al Lawati, and Mohammed M. Bait- Suwailam, "Design and Implementation of a Vehicle to Vehicle Communication System Using Li-Fi Technology", International Conference on Information and Communication Technology Research (ICTRC), 2015.

2. Pooja Bhateley, Ratul Mohindra, S.Balaji, "Smart Vehicular Communication System Using LI-FI Technology", International Conference on Computation of Power, Energy Information and Communication (1CCPEIC), 2016.

3. Harald Haas ; Liang Yin ; YunluWang ; Cheng Chen, "What is Li-Fi", Journal of Lightwave Technology (Volume: 34, Issue: 6, March $15,2016)$.

4. N. G. Ghatwai, V. K. Harpale and M. Kale, "Vehicle to vehicle communication for crash avoidance system," 2016 International Conference on Computing Communication Control and automation (ICCUBEA), Pune, 2016, pp. 1-3. doi: 10.1109/ICCUBEA.2016.

5. J. Lianghai, M. Liu, A. Weinand and H. D. Schotten, "Direct vehicle-tovehicle communication with infrastructure assistance in 5G network," 2017 16th Annual Mediterranean Ad Hoc Networking Workshop (MedHoc-Net), Budva, 2017, pp. 1-5. doi: 10.1109/MedHocNet.2017

6. Isamu Takai, Member, IEEE, Tomohisa Harada, Michinori Andoh, Keita Yasutomi, Member, IEEE, Keiichiro Kagawa, Member, IEEE, Shoji Kawahito, Fellow, IEEE "Optical Vehicle to Vehicle Communication System Using LED Transmitter and Camera Receiver", IEEE Photonics Journal, DOI 10.1109/JPHOT.2014.2352620, 2014.

7. K. Vijayakumar and C. Arun, "Continuous Security Assessment of Applications in Cloud Environment", International Journal of Control Theory and Applications, ISSN: 0974-5645 volume No. 9(36), Sep 2016, Page No. 533-541.

8. R.Joseph Manoj, M.D.Anto Praveena, K.Vijayakumar, “An ACO-ANN based feature selection algorithm for big data", Cluster Computing The Journal of Networks, Software Tools and Applications, ISSN: 1386 7857 (Print), 1573-7543 (Online) DOI: 10.1007/s10586-018-2550-z, 2018.

9. K. Vijayakumar and V. Govindaraj, "An Efficient Communication Technique for Extrication and Cloning of packets on cloud", International Journal of Applied Engineering Research, ISSN 0973-4562 Vol. 10 No.66 May 2015 


\section{AUTHORS PROFILE}

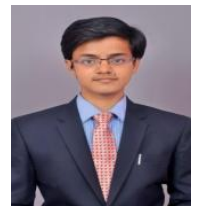

Rehan Ahmad Khan was born on 19th July, 1996 in the holy city of India, Varanasi. He completed his B.Tech. in Electronics and Communication course from SRM INSTITUTE OF SCIENCE AND TECHNOLOGY, KATTANKULATHUR. His paper titled "Health monitoring system in Robotic wheelchair with internet of things" got published in International Journal of Science and Innovative Engineering and Technology (IJSIET), Vol.3, May 2018. He has been active member of IEEE think tank team of SRM (student branch) during his college days. Being a member of think tank, he organized National level Entrepreneurship summit - Alpha summit at SRM. During his stay at SRM, he had done various projects such as Lost plane finder, electromagnetic lock and many more. The paper presented in this volume is the final year project of the author, which got accolodates from SRM for presentation in department COMSPRO exhibition. He has couple of hands on industrial experience. One in defence enterprise, Bharat Electronics Ltd. and other being energy enterprise of India, Oil and Natural Gas Corporation Ltd. Former in the field of quality, testing \& development and engineering department. Later, in the field of electronics specialization used in locating and extracting oil resources from reservoirs deep beneath the earth.

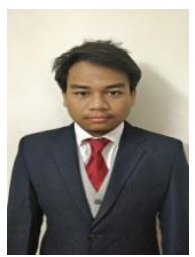

Agastya Gogoi was born in 22nd December of 1996. He has had a love affair with science mainly electronics for as long as he can remember. Since he was a kid in school, he has known to be wanting to be a scientist. He is always dreamed about working as an electronics Scientist in NASA. He completed his bachelor's degree from SRM, KATTANKUTHUR. He has done many projects while in SRM, including automobile detection and prevention system and automatic gate and so on. The project involving automobile detection uses Lifi and Wi-Fi as its base of mechanism. The subject of electronics has excited Agastya to an extent as to take it as a subject for masters. He has worked as an intern in IBM and in heavy industry companies like Oil India Limited in Noida. He has had an weird urge to learn everything about microcontrollers and its components and the idea of linking both software and hardware to make a circuit work.

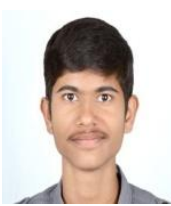

Rahul Srivastava was born in Lucknow, India. He received his Bachelor's degree in Electronics and Communications Engineering in 2019 for SRM Institute of Science And Technology, Kattankulathur. His main area of research in the final major project titled "Automobile Collision Warning and Identification system using visible light and Wi-Fi communication" was the use of Li-Fi to share data between vehicles at rapid speed. He has also done research on photonic optical fiber cable to increase throughput. He currently is employed in Infosys as a system engineer. His current interests lie in innovative Technology in the field of photonics and optoelectronics which are beneficial to society

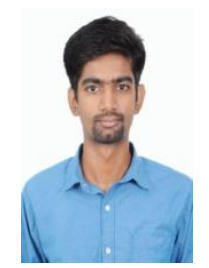

Shubham Kumar Tripathy was born in Sonepur, Bihar, India. He did his schooling from KendriyaVidyalaya. He received his bachelor's degree in Electronics and Communications Engineering in 2019 from SRM Institute of Science and Technology, Kattankulathur. He is an avid programmer and loves to meditate in his free time. He likes sketching and reading spritual books. In his major project titled "Automobile collision Warning And Identification system using visible light and Wi-Fi communication" his main research area was the communication between the Li-Fi module and the mobile. He has also made the mobile application for the same. He did an internship in Hawlett Packard Enterprise in electronics domain for 1 month. He has also worked in a startup called Enermaze sports Pvt LTD for 2 months. He is currently employed at Infocepts in the designation of Associate Analyst.

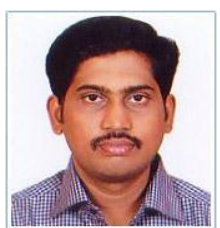

Mr.S. Manikandaswamyis working as an Assistant professor at SRM Institute of science and technology, Chennai since 2007. He obtained his bachelor degree in B.E ( ECE ) from Madras University and Master degree (M.Tech) from SASTRA Deemed University. Currently he is Pursuing his Doctoral degree at SRM Institute of science and technology. He has published papers in national and international journals and participated in various workshops and conferences. His area of interest are Visible light communication, Optical communication, Wireless communications and secure transmission in Wireless communication. Currently he is working on modulation techniques for high speed data in Visible light communication. 